\title{
Expresión de tres perspectivas de la justicia social en la organización de los sistemas de salud*
}

\section{Expression of three perspectives of social justice in the organization of health systems}

\section{Expressão de três perspectivas da justiça social na organização dos sistemas de saúde}

Fecha de recepción: 02-06-15 Fecha de aceptación: 04-09-15 Disponible en línea: 01-11-15

doi:10.11144/Javeriana.rgyps14-29.etpj

Cómo citar este artículo:

Giraldo-Piedrahita F. Expresión de tres perspectivas de la justicia social en la organización de los sistemas de salud. Rev. Gerenc. Polít. Salud. 2015; 14(29): 26-40. http://dx.doi.org/10.11144/Javeriana. rgyps14-29.etpj

Artículo de reflexión

** Médico y cirujano de la Universidad de Antioquia, magíster en Salud Pública de la Facultad Nacional de Salud Pública "Héctor Abad Gómez", Universidad de Antioquia. Actualmente es estudiante de Doctorado en Salud Pública en la 26 Facultad Nacional de Salud Pública "Héctor Abad Gómez" de la Universidad de Antioquia, donde se desempeña como docente en el departamento de Ciencias Específicas. Correspondencia: Medellín, Calle 62 No 52-59, Oficina 301. Teléfono 21968 84. Correo electrónico: fgiraldopi@gmail.com 


\section{Resumen}

Este artículo se aproxima al asunto de las perspectivas filosóficas de la justicia social y su relación con algunas de las implicaciones en los sistemas de salud en Latinoamérica y en el mundo. Se revisan tres perspectivas filosóficas de justicia social, desde el enfoque liberal radical, el liberal igualitarista o redistributivo y el marxista o igualitarismo. A partir de estos enfoques se identifican los soportes teóricos, la relación Estado-sociedad, sus concepciones de salud y el enfoque de salud pública. Con base en estos conceptos se identifican algunas implicaciones para los sistemas de salud en cada una de sus vertientes representativas. El ensayo termina con unas reflexiones alrededor de la necesidad de debatir el derecho fundamental a la salud, como un elemento fundante de los sistemas universales de salud y se asume que aporta una perspectiva para el diseño y análisis de sistemas de salud desde el derecho a la salud.

Palabras clave: justicia social; sistemas de salud; salud pública; derecho a la salud; acceso universal a servicios de salud; políticas públicas de salud

\section{Abstract}

This article approaches the issue of philosophical perspectives of social justice and its relationship with some of the implications for health systems in Latin America and the world. Three philosophical perspectives of social justice from the radical liberal approach, egalitarian and redistributive liberal, and Marxist egalitarianism or reviewed. Society, their conceptions of health and public health approach. From the theoretical support these approaches, the relationship State are identified. Based on these concepts identify some implications for health systems in each of its representative aspects. The paper concludes with some reflections about the need to discuss the fundamental right to health as a fundamental element of universal health systems and assume a perspective as input for the design and analysis of health systems from the right to health.

Keywords: social justice; health systems; public health; right to health; universal access to health care services; public health policy

\section{Resumo}

Este artigo aproxima-se do assunto das perspectivas filosóficas da justiça social e sua relação com algumas implicações nos sistemas de saúde em Latino-américa e o mundo. Revisam-se três perspectivas filosóficas de justiça social, desde o enfoque liberal radical, o liberal igualitarista ou redistributivo e o marxista ou igualitarismo. A partir destes enfoques identificam-se os suportes teóricos, a relação Estado-sociedade, suas concepções de saúde e o enfoque de saúde pública. Com base nestes conceptos identificam-se algumas implicações para os sistemas de saúde em cada uma das suas vertentes representativas. O ensaio termina com reflexões ao redor da necessidade de debater o direito fundamental à saúde, como elemento fundante dos sistemas universais de saúde e assume-se que contribui com uma perspectiva para o desenho e análise de sistemas de saúde desde o direito à saúde.

Palavras-chave: justiça social; sistemas de saúde; saúde pública; direito à saúde; acesso universal a serviços de saúde; políticas públicas de saúde 


\section{Introducción}

El debate alrededor de la justicia social y sus alcances en los sistemas de salud es muy importante hoy para la salud pública, porque, siguiendo a Vega, ayuda a comprender cómo "los alcances de la justicia sanitaria están en estrecha relación con la lógica del diseño de un modelo económico, social y de salud, y con la praxis que ésta determina entre sus actores" (1).

Aunque la complejidad de este debate supera considerablemente las posibilidades de esta reflexión, se parte de retomar algunas ideas ordenadoras que han orientado las posiciones alrededor de la justicia social.

Es fundamental comprender que los sistemas de salud tienen orígenes y dinámicas que corresponden a lógicas provenientes en gran parte de la concepción de justicia social dominante en cada momento histórico. Entonces, este ensayo pretende identificar algunos elementos relacionados con el concepto de justicia social que influyen en la organización y desempeño de los sistemas de salud, con el fin de realizar un análisis más profundo y coherente a estos, y poder dilucidar la estructura de las organizaciones y sus dinámicas, la selección de los modelos operativos y los elementos para realizar las evaluaciones de los sistemas de salud y su impacto en las poblaciones. Es así como en el ensayo se escogen tres corrientes, ellas son: la liberal radical u ortodoxa, la liberal igualitarista o redistributiva y la marxista o igualitarismo, y a partir de estas se identifican algunas de las implicaciones que se han presentado en los sistemas de salud, para luego proponer unas reflexiones alrededor de algunos elementos para el diseño y análisis de sistemas de salud desde el derecho a la salud, asumiendo este como un derecho humano fundamental y transversal de los sistemas de salud.
Es necesario hacer explícito que no se considera que existan aplicaciones puras para las tres perspectivas de justicia social y sus alcances en los sistemas de salud; más bien se cree que en los contextos reales se ven tendencias y mixturas en sus estructuras, en los principios filosóficos y en su funcionamiento. Es así como se asume que una mirada a otras posturas concretas permite la emergencia de contrastes y elementos críticos de utilidad a los debates actuales acerca de los sistemas de salud en el mundo, la cobertura universal y su papel en el logro de la equidad en salud.

\section{Perspectiva filosófica de justicia social desde el enfoque liberal ortodoxo o liberal radical}

\section{Elementos teóricos y relación Estado- sociedad}

Desde el enfoque liberal ortodoxo o también llamado radical, hay por lo menos dos consensos básicos alrededor de lo justo: el derecho a la vida y el derecho a la libertad, los cuales forman parte de los llamados derechos humanos de primera generación. El respeto por estos derechos se encuentra en la base de la formación de los Estados modernos, del pensamiento ilustrado y de las revoluciones liberales; asimismo, regulan la relación Estado-ciudadano. Las posturas liberales adicionalmente han defendido un tercer derecho, el de la propiedad privada, como producto del trabajo, la herencia o las transacciones entre personas libres $(2,3)$.

La igualdad para esta corriente se refiere solo a la condición natural de hombres libres, sin trascender otros ámbitos. Hernández a este respecto señala que "Si hubiera alguna redistribución en favor de los que no tienen propiedad (los pobres) sólo sería como un acto voluntario de caridad o de beneficencia, 
a partir de un sentimiento de compasión de los propietarios, pero no en función de la justicia” (4). Es así como la justicia se materializa en las transacciones justas entre hombres libres e iguales, las cuales se perfeccionan a través de contratos (5).

Estas consideraciones implican que el Estado no debe intervenir en otros asuntos, pues los sujetos son libres y autónomos y deben garantizarse por sí mismos las condiciones para la vida que desean vivir. Así, la búsqueda de la satisfacción de las necesidades y aspiraciones individuales debería conducir al bienestar colectivo. Para los representantes del pensamiento liberal radical, la intervención del Estado conduce de una sociedad libre a una autoritaria, y es el mercado, en forma imparcial, el llamado a dirimir los problemas de distribución (6-8).

Entonces, la interacción de la sociedad con el Estado no se da en términos del ejercicio de la ciudadanía de los actores políticos, sujetos de derechos y deberes, sino como usuarios o clientes individuales o colectivos que esperan satisfacer sus demandas de atención en el ámbito del mercado, por medio de relaciones contractuales que deben ser protegidas por el Estado (9).

\section{Concepto de salud y enfoque de salud pública}

Desde esta perspectiva, el concepto de salud enfatiza en las dimensiones biológicas antes que en las socioculturales, excluyendo a estas últimas de su papel en la generación de la enfermedad y por ende de las respuestas que pueda dar la sociedad en este campo. Esta se fortalece con el modelo hospitalario, que mantiene su hegemonía durante el siglo xx, con base en la atención de la enfermedad particular de los individuos (10).
Así, esta concepción se apoya en el método positivista para explicar el riesgo de enfermar en la población. De esta manera, la epidemiología ha desarrollado la idea de la salud y la enfermedad como un equilibrio biológico o energético-material, de expresión individual y con causas internas o externas identificables como "factores", con apoyo en la causalidad probabilística y factorial de la epidemiología multicausal (11).

En la década de los noventa, las políticas de salud en el continente estuvieron marcadas por las propuestas de reformas del sector salud, ampliamente promovidas por organismos financieros multilaterales. En ese sentido, es pertinente enfatizar que esas reformas no fueron procesos aislados, ya que formaron parte de procesos más amplios de reforma del Estado, los cuales conocimos como ajustes estructurales y se desarrollaron a partir de las propuestas generadas en el asî llamado "consenso de Washington", el cual consistió en un conjunto de medidas de política económica generadas por una reunión de expertos del Fondo Monetario Internacional (FMI), el Banco Mundial, la Reserva Federal de los Estados Unidos y el Congreso de ese país, realizado en Washington en 1989, cuyo propósito fundamental era elaborar un conjunto de propuestas para incorporar a todos los países del "mundo en desarrollo" al mundo del mercado (12).

Con base en los elementos teóricos expuestos, se deduce que no existe justificación moral para considerar la salud como derecho humano y, por tanto, tampoco constituye un deber del Estado, por el contrario "los servicios deben ser obtenidos en la libre competencia de mercado y capacidad de pago y en el marco de las transacciones justas, expresadas en contratos garantizados por el Estado" (13). Es decir, en una lógica de bienes privados, en donde la salud de las personas puede entenderse como mercancía, limitada 
en su calidad y cantidad por la capacidad de pago y no ajustada a la necesidad (9).

Es así como se configura una visión de la justicia de tipo individualista, ligada a la propiedad y a la distribución individual y focalizada de recursos públicos o de filantropía privada (14). Esta postura divide la salud pública en dos: la prestación individual de servicios y bienes para mejorar el nivel de salud de las personas, prestación que debe ser provista desde la lógica del intercambio comercial, y por otro lado, la minimización de papel del Estado, al que se le asigna como responsabilidad la provisión de servicios altamente costo-efectivos y con altas externalidades positivas (la inmunización de un niño disminuye la transmisión del sarampión y otras enfermedades, lo que confiere a esa medida una externalidad positiva). Esta es la perspectiva hegemónica que ha guiado las políticas sociales y de protección social en muchos países de Latinoamérica desde la década de los años ochenta y noventa del siglo pasado $(15,16)$.

En este sentido, Granda define algunas características de este enfoque de salud pública al que denomina "enfermología pública", estas son: el presupuesto filosófico-teórico de la enfermedad y la muerte como punto de partida para la explicación de la salud; el método positivista para explicar el riesgo de enfermar en la población y el estructuralfuncionalismo para comprender la realidad social y el reconocimiento del poder del Estado como fuerza privilegiada para asegurar la prevención de la enfermedad (17).

Es así como estas características han servido de instrumento para la toma de decisiones en términos de focalización de recursos, cálculo de los paquetes de prestación de servicios, planes de atención básica, estudio de las medidas de reducción del gasto en salud y cálculos de subsidio a la demanda, lo cual se traduce en individualización de la salud y focalización de la enfermedad $(13,18)$.

\section{Implicaciones para los sistemas de salud}

Para precisar el concepto de sistema de salud, esta postura recoge la de la Organización Mundial de la Salud (oms), la cual sirve como introducción para lo que se presenta en los párrafos siguientes, que lo define como el conjunto de organizaciones, individuos y acciones cuya intención primordial es promover, recobrar o mejorar la salud. Esto incluye las acciones dirigidas a incidir en los determinantes de la salud, así como las orientadas a mejorar la situación sanitaria de la población (19). A su vez, se definen como funciones principales de los sistemas de salud, la provisión de servicios, la generación de recursos, el financiamiento y la rectoría. Asimismo, se enfatiza en lograr niveles óptimos de salud y eliminar las inequidades en el acceso, como el objetivo fundamental de estos (20). Es importante aclarar que en el desarrollo del texto se discuten distintas perspectivas de la justicia social que se expresan en los sistemas de salud en la concepción dominante de la salud, el derecho social o bien privado individual, el modelo de atención que define la organización de los servicios: integral o fragmentado, básicamente dedicado a la atención de la enfermedad, el tipo y las fuentes de financiamiento del sistema, el papel de los ciudadanos en su organización y control y los resultados obtenidos en términos de salud de la población.

\section{Formas de organización-financiamiento.}

Desde esta perspectiva de justicia social, las formas de organización y estructuración de los sistemas de salud se dan con base en el mercado de seguros, en la división de lo público y lo privado, en la división Estadomercado, en la cotización individual de los usuarios del sistema y en el subsidio a la demanda para los que demuestran que 
no tienen capacidad de pago (13). De esta manera, se defiende el libre mercado como el escenario adecuado para el acceso a los servicios de salud (16).

La organización del modelo es descentralizada y con poblaciones segmentadas según ingresos y capacidad de pago, lo que se ve reflejado en la fragmentación de los servicios y en la ausencia de integralidad en la atención (21).

Prestación de los servicios. Este enfoque privilegia que los recursos para la salud se dediquen a la prestación de servicios de salud individuales, especialmente de diagnóstico, tratamiento y rehabilitación, y a algunas acciones de protección específica, lo que ha motivado que se incentiven presiones financieras, el ánimo de lucro y el eficientismo en la conducta de los profesionales y de las instituciones de salud (1,22). A su vez, debido a que los mercados privados por sí solos proporcionan muy pocos de los bienes públicos fundamentales para la salud, se requiere participación gubernamental (acciones en salud pública) para incrementar el suministro de esos bienes. Estos servicios de salud tienen grandes externalidades, lo que quiere decir que su utilización por un individuo beneficia a los demás miembros de la sociedad (las inmunizaciones en los niños) (23).

De este modo, se incentiva el predominio del criterio economicista de las actividades asistenciales, sin reconocer las contingencias humanas, sociales y culturales propias de estas, incidiendo en la probabilidad de que se disminuya la calidad de la atención al proteger el ingreso per cápita de las aseguradoras, sacrificando la calidad por la eficiencia. Así, las empresas no incurren en pérdidas ni en disminución de la rentabilidad (9). Además, se promueven las formas de selección adversa por parte de las aseguradoras, con la implementación de barreras de acceso para ciertos grupos de edad (ancianos, menores de un año), cierto tipo de enfermos (con SIDA, enfermedades renales crónicas, con cáncer, enfermos mentales), o que requieren determinados tipos de servicios (unidad de cuidados intensivos, salud mental o salud oral) (9).

Participación ciudadana. Como ya se mencionó, la participación ciudadana en los sistemas de salud, no se da en términos del ejercicio de la ciudadanía de los actores políticos, sujetos de derechos y deberes civiles, sino como usuarios o clientes individuales o colectivos que esperan satisfacer sus demandas de atención. Así, se produce una participación individualista en calidad de consumidor en el mercado de servicios, pero no como un actor sociopolítico, y se puede afirmar que ha sido el triunfo de la hegemonía individualista y consumista que destituye los derechos colectivos, se centra en los derechos de propiedad (contratos y seguridad) y justifica el asistencialismo $(4,9)$.

\section{Perspectiva filosófica de justicia social desde el enfoque liberal igualitarista - liberal redistributivo}

\section{Elementos teóricos y relación Estado- sociedad}

Desde esta perspectiva, se parte de un reconocimiento básico de la fundamentación moral de los derechos humanos en relación con el concepto kantiano de persona o "sujeto moral" de todo ser humano, como una categoría universal que fundamenta la autonomía y la autodeterminación, es decir, la libertad humana. Esta condición de "sujeto moral" es la mejor expresión de la igualdad y sustenta la idea de "dignidad" (24). En este mismo sentido, el pensamiento de Kant orienta la concepción de los hombres como 
sujetos morales que desean vivir en una sociedad justa y ordenada, construida en el ámbito del Estado-nacional (25).

Rawls, por su parte, retoma la perspectiva kantiana y plantea que la idea de libertad no es realizable en el marco de sociedades inequitativas, y se preocupa por la justicia distributiva. Es así como considera que para el logro de la libertad y la autonomía se requiere garantizar a las personas unas condiciones básicas que permitan dicho ejercicio en el marco de una democracia deliberativa. Estas condiciones deben desarrollarse a partir de la construcción de un consenso social en los Estados nacionales; es decir, el debate se inscribe en términos de la equidad nacional (25). Así, la ampliación de la ciudadanía a través de los derechos implica el reconocimiento de la relación estructural entre los valores de "libertad, igualdad y dignidad" (24). En este sentido, los derechos sociales se justifican en la medida en que posibilitan la autonomía y el disfrute efectivo del derecho a la libertad y el desarrollo de la vida que se quiere vivir (25).

Precisamente, es en la perspectiva redistributiva de la justicia en la que se apoya la Comisión de los Determinantes Sociales de la Salud (CDSs), la cual es una combinación entre el principio de libertad y el de igualdad, que considera la complementariedad entre la "libertad de ser", entendida como el derecho negativo liberal que el Estado debe proteger (libertad, vida), con la "libertad para ser", entendida como las condiciones y medios para ejercer la libertad y que constituyen los derechos positivos económicos, sociales y culturales (26).

Con base en esta visión de lo justo, la condición de ciudadano generó una obligación de los Estados de proveer, con pretensión universal, condiciones de vida dignas, expresadas en trabajo, educación, saneamiento, asistencia médica, vivienda, etc. $(27,28)$. Esta concepción lleva a que el énfasis de la ruta transformadora esté puesto sobre las políticas públicas redistributivas, desde la organización estatal existente y con mecanismos de participación, en el sentido de la "gobernanza", con ejercicios de "empoderamiento" de los afectados por las inequidades y el logro concertado de un compromiso de responsabilidad social de las organizaciones privadas, tal como aparece en la convocatoria de la cDSs (29).

\section{Concepto de salud y enfoque de salud pública.} El concepto de salud, desde esta perspectiva, se fundamenta en las investigaciones que mostraron un gradiente notable de mortalidad entre los grupos socio-ocupacionales de la sociedad inglesa. El Whitehall Study, de Marmot et al., sobre las diferencias en mortalidad cardiovascular entre empleados públicos según la jerarquía burocrática, ratificó algo que se había discutido en Europa, en especial desde el siglo xIX: el origen social de la salud y la enfermedad humana (29). Usando la posición socio-ocupacional, los autores mostraron las desigualdades en los resultados en salud relacionadas con la posición social de las personas (30).

En este sentido, la cDss afirma que "la mala salud de los pobres, el gradiente social de salud dentro de los países y las grandes desigualdades sanitarias entre los países están provocadas por una distribución desigual, a nivel mundial y nacional del poder, los ingresos, los bienes y los servicios, y por las consiguientes injusticias que afectan a las condiciones de vida de la población de forma inmediata y visible (acceso a atención sanitaria, escolarización, educación, condiciones de trabajo y tiempo libre, vivienda, comunidades, pueblos o ciudades) y a la posibilidad de tener una vida próspera. Esa distribución desigual de experiencias perjudiciales para la salud no es, en ningún caso, un fenómeno 
natural. Los determinantes estructurales y las condiciones de vida en su conjunto constituyen los determinantes sociales de la salud" (29).

En el informe de la cDss/oms se define una propuesta de relaciones jerárquicas entre los determinantes sociales, económicos y políticos de la salud, denominados "determinantes estructurales" (gobierno, políticas macroeconómicas, políticas sociales, políticas públicas, valores sociales y culturales), los cuales están ligados al concepto de "posición social" de las personas, representado en la posición socioeconómica, el género, la etnia y la raza. De estos determinantes se derivan los denominados "determinantes intermedios de la salud", esto es, las condiciones en que viven y trabajan las personas, la disponibilidad de alimentos, los factores biológicos, los comportamientos y los factores psicosociales, que generan exposiciones, daños y respuestas diferentes. Finalmente, ambos, estructurales e intermedios, impactan con resultados desiguales en la salud y en el bienestar de la población. Estas diferencias se plantean como consecuencia de la estratificación social, la cual, a su vez, se produce por una distribución desigual del poder, los ingresos, los bienes y los servicios (29,31).

Dentro de las implicaciones prácticas desde la cDss está resolver las inequidades (subsanar las desigualdades), incrementando el nivel de quienes están en una situación desfavorable, para llevarlos al nivel de los más saludables (32). El responsable fundamental es el Estado, no obstante, se reconoce el empoderamiento de las personas y comunidades de su salud y los factores que la determinan (29). Esta concepción redistributiva de la CDSs está soportada en una ideología socialdemócrata y en el principio de justicia distributivo, ya que propone impactar las inequidades por medio de la redistribución de los bienes y los medios. La salud, en este sentido, se concibe como derecho de los ciudadanos y, asimismo, se propone una cobertura universal para la población, donde los beneficios parten de un mínimo vital y la administración y la organización es públicoprivada (21).

\section{Implicaciones para los sistemas de salud}

Formas de organización-financiación. Desde este enfoque, el aseguramiento universal "surge de una concepción individual, donde cada persona debe afiliarse y cotizar o demostrar su condición de pobreza para recibir un subsidio para el pago de las primas" (33). Esto se ve reflejado en la organización de los modelos de sistemas de salud, que se caracterizan por una universalidad constituida por la segmentación poblacional y la capacidad de pago de las personas.

De este modo, el sistema se fundamenta en una relación Estado-mercado, a través de un Estado interventor. Sin embargo, se ha criticado que esta función no se haya cumplido a cabalidad, por la débil posición de los entes estatales frente a otros actores, fortalecidos económicamente (aseguradoras, multinacionales) y con gran poder de negociación. Igualmente, el sistema, al apoyarse en modelos de mercados segmentados de acuerdo con el poder adquisitivo, ha generado barreras de acceso económicas, administrativas y socioculturales que impiden una atención integral a los usuarios (4).

De esta manera, el financiamiento se procura por fuentes separadas, con base en los impuestos de los ciudadanos, cotizaciones de empleados y empleadores y gastos de bolsillo de los usuarios del sistema, sin perder de vista en este punto que estos últimos (gastos de bolsillo) afectan el ingreso base familiar de las familias y por ende disminuyen la 
posibilidad de proveerse otras necesidades básicas (4).

Prestación de los servicios. Desde esta perspectiva, "la cobertura poblacional universal es el resultado de la suma de diversos modelos de aseguramiento, con coberturas parciales de servicios, que varían en cada país dependiendo de factores tales como aportes y nivel de renta de los grupos poblacionales, carga de enfermedad, costo beneficio, etc.". De esta forma, el aseguramiento universal, en general no pretende el cubrimiento de todas las prestaciones sino que se enfoca en una serie de paquetes básicos, denominados pisos mínimos, "diferenciados conforme al aporte o nivel de ingreso de los grupos poblacionales, por lo que la universalidad, en este caso, no se concretiza" (33).

En este sentido, "el aseguramiento tiende a concentrar los servicios en la atención individual y en áreas urbanas y no como una verdadera red presente en el territorio, descuidando las labores de la salud pública, de la promoción de la salud y la acción sobre su determinación social” (33).

Participación ciudadana. Como ya se dejó entrever en la relación Estado-sociedad, desde esta perspectiva, el responsable fundamental de impactar las inequidades en salud y mejorar las condiciones sanitarias es el Estado; no obstante, se reconoce el empoderamiento de las personas y comunidades de su salud y los factores que la determinan, además, se plantea el logro concertado de un compromiso de responsabilidad social de las organizaciones privadas (29). Este empoderamiento ciudadano y el compromiso privado representan un avance con relación a la postura liberal radical, ya que de una manera más determinante se busca el logro efectivo del disfrute a la salud como un derecho, elemento ausente en la perspectiva liberal radical.

\section{Perspectiva filosófica de justicia social desde el enfoque marxista - igualitarismo}

\section{Elementos teóricos y relación Estado- sociedad}

Desde esta perspectiva, una sociedad justa se regiría por la máxima de Louis Blanc, adoptada por Marx: "a cada uno debe exigírsele según su capacidad, y debe dársele según sus necesidades" (26).

Hernández amplia el concepto, planteando que "las desigualdades son injustas porque son producidas por una apropiación indebida por parte de una clase, un género o una etnia, de los medios de realización de la vida de las personas" (15). Además, con base en la máxima de Blanc, refiere que "Sólo si se afectan las condiciones en las cuales se produce una distribución desigual del poder en la sociedad, en función de la propiedad de los medios, o de los privilegios acumulados de género o de raza, se podrá construir una sociedad justa, en función de las desiguales capacidades y necesidades humanas" (15).

Quevedo y Hernández hacen énfasis, desde esta perspectiva, en el concepto de "sujeto histórico", constituido como tal por las relaciones sociales y sujetado a la sociedad a la que pertenece, siempre en capacidad de transformar su realidad (34). A su vez, desde el punto de vista de Breilh, resulta un "sujeto político" transformador de su realidad, con capacidades y prácticas emancipadoras orientadas a superar las múltiples enajenaciones que resultan de las relaciones de poder existentes" (18).

En este sentido, siguiendo a Breilh, se entiende la inequidad como las desigualdades producidas por las relaciones de poder, de dominación y resistencia, con múltiples ex- 
presiones y con tres ejes estructurales que se articulan de manera compleja en el modo de producción capitalista y configuran lo que el autor ha denominado "la triple inequidad": de clase, de género y de etnia (18).

\section{Concepto de salud y enfoque de salud pública}

Para aclarar el concepto de salud de acuerdo con este enfoque, se debe recurrir a la Medicina Social Latinoamericana (MSL) y a la Salud Colectiva (sc), que presentaron la idea de "proceso" salud-enfermedad, de naturaleza social y por tanto histórico, con expresión específica en el "nexo biopsíquico humano" e inmerso o "subsumido" en los procesos de producción y reproducción social $(35,36)$.

Breilh desarrolla el concepto de salud desde lo multidimensional: "[...] se la puede dimensionar tanto como objeto (dimensiones ontológicas), cuanto como concepto (dimensiones propiamente epistemológicas), así como campo de acción (dimensiones práxicas)". Además, amplia el concepto de proceso y ubica la salud en las dimensiones general, particular y singular, “[...] Como proceso se realiza en la dimensión general de la sociedad, en la dimensión particular de los grupos sociales y en la dimensión singular de los individuos y su cotidianidad". En el terreno de la intervención también puede trabajarse en la dimensión curativa, preventiva y de promoción, en tanto que en lo que atañe a lo epistemológico, la salud puede mirarse desde la perspectiva de diversas culturas, lo cual implica el respeto a las diferencias culturales y la voluntad para que ellas sobrevivan, es decir, un proceso de construcción conjunta mediante la integración de varias culturas, en medio de una visión solidaria, de respeto a las diferencias culturales bajo equidad de derechos y oportunidades, a la vez que continúen y se fortalezcan las culturas, en un enriquecimiento mutuo. Significa, entonces, un movimiento que respeta y construye los vínculos y encuentros, los valores democráticos solidarios compartidos por varias culturas y la defensa de los espacios e instituciones que hacen viable esa construcción (18).

Desde esta perspectiva, se hace énfasis en la identificación de los movimientos de generación y reproducción, de deterioro y protección, de desgaste y recuperación, "modo de vida". Pero el modo de vida de un grupo social no obedece únicamente a su propia historia, sino que está relacionado además con la historia más amplia y general de la sociedad, y a su vez el modo de vida no solo se expresa en estilos de vida de las personas y su cotidianidad, sino que lo hace en los procesos que ocurren en los organismos de estas. Esa determinación no va en un solo sentido, ya que estos fenómenos más específicos también llegan a expresarse en las instancias más generales (18), en complejidades que van de la dinámica general, particular y singular del proceso salud-enfermedad humano. "Más que ex-posición a riesgos externos, los sujetos sufren procesos de imposición de condiciones de vida" (37).

Esta concepción de lo justo, sumada a la concepción de salud antes descrita, siguiendo a Borrero "ha permeado las corrientes de la medicina social y la salud colectiva en América Latina, las cuales han promovido la salud como derecho humano fundamental articulado al desarrollo de condiciones de vida y de trabajo que dignifiquen al ser humano, superen la cosificación producida por el capital y la garantía del acceso efectivo a servicios de salud integrales, adecuados y suficientes de acuerdo con las necesidades de los diferentes grupos humanos" (6).

\section{Implicaciones para los sistemas de salud}

Formas de organización y financiación. Una pretensión importante, desde esta pers- 
pectiva, es el logro de sistemas universales de salud. Estos nacen de una concepción colectiva y de derecho ciudadano, del derecho humano a la salud (33). El derecho a la salud se entiende, en forma general, desde la visión del Instituto Suramericano de Gobierno en Salud (ISAGS), "como el derecho a la atención a la enfermedad, así como el cuidado del bienestar físico y mental de las personas, sin perder de vista que el derecho a la salud forma parte del derecho a la vida, comprendida ésta, en toda la dimensión de la existencia humana dentro de un espacio que va más allá de lo individual: la persona en su entorno familiar, comunitario, como parte de la naturaleza y el universo" (33).

Desde su parte organizativa, estos sistemas universales se caracterizan por una modalidad "con rectoría del Ministerio de Salud e intervención de las tres esferas territoriales del Estado (nacional, departamental, municipal) para integrar todas las funciones del sistema, que van desde la definición de políticas hasta la prestación de servicios, garantizando desde la promoción hasta la rehabilitación de la salud" (33).

El financiamiento en el sistema universal de salud se sostiene con las contribuciones de toda la sociedad a través de sus impuestos (sobre la renta y el consumo), que van al tesoro general de la Nación. Es un sistema solidario, donde el acceso a los servicios es determinado por las necesidades en salud y no por el monto de la contribución individual (1).

Prestación de los servicios. En los sistemas universales de salud la cobertura es total, tanto en términos de población como en términos de servicios, no clasifica ni restringe, pues todo lo que existe en el sistema está disponible para todos, siguiendo, por supuesto, criterios de racionalidad basados en las necesidades de las personas y definiendo claramente los me- canismos y puertas de entrada para el acceso a cada nivel de atención (33).

La integralidad se debe destacar como un rasgo que debe caracterizar a los sistemas universales de salud. Para Vega, "Se concibe la integralidad, como esa dimensión que da cabida a los cursos de la vida, a la interculturalidad, al rescate de la medicina tradicional de los pueblos, junto con procesos para mejorar la calidad y la organización de los servicios de salud en verdaderas redes, desde el espacio comunitario y familiar, hasta los centros de especialización” (1).

Participación ciudadana. Dentro de las funciones de los sistemas universales de salud está la de garantizar el derecho a la salud, con participación social, interculturalidad, solidaridad y acción sobre la determinación social de la salud (38). Se debe tener presente que, desde esta concepción filosófica de la justicia, "no se espera una transformación del estado de cosas prioritariamente desde el poder estatal, sino desde la intersubjetividad emancipadora entre las clases, los géneros y las etnias dominadas" (18). Lo que requiere un real empoderamiento con relación a los derechos y deberes por parte de los ciudadanos para poder reclamar, asumir y gozar en forma efectiva del derecho a la salud en su connotación de fundamental.

\section{Elementos para el diseño y análisis de sistemas de salud desde el derecho a la salud}

La salud como derecho humano fundamental: elemento transversal de los sistemas de salud

Según el recorrido teórico presentado hasta el momento, es pertinente aclarar que los modelos económicos, las dinámicas sociales y 
los sistemas de salud están en relación directa con los alcances de la concepción de justicia sanitaria, y por ende con la interacción y el desempeño de sus actores (1).

En este sentido, es innegable que el posicionamiento de las políticas neoliberales ha influido considerablemente en la organización de sistemas de salud basados en el mercado, apoyados en un enfoque liberal de la justicia social. Pero a su vez, ha venido emergiendo con solidez el discurso de los derechos humanos, con el fin de avanzar en el mejoramiento de las condiciones de vida de la población, soportado en las corrientes de justicia social distributiva y desde el igualitarismo (6).

Entonces, es prioritario introyectar y convertir en la columna vertebral de los sistemas de salud la reivindicación de la salud como derecho humano fundamental. En este sentido, la definición de la salud como derecho humano fundamental implica acoger y traducir en políticas públicas las características y los principios rectores de los derechos humanos: universalidad y accesibilidad en la asistencia sanitaria para todos, sustentada en la condición de ser humano; indivisibilidad e interdependencia, que supone el reconocimiento, en la práctica, de la salud como componente del bienestar y la integralidad de las políticas sociales; dimensión individual y colectiva, porque la salud individual depende y afecta a otros, y porque la prestación de servicios debe garantizar la asistencia individual y la salubridad pública; progresividad e irreversibilidad, que implica una inversión estatal sistemática en la vía de la universalidad y el no retroceso en los logros alcanzados; irrenunciabilidad, porque ningún ser humano puede renunciar a sus derechos fundamentales y ninguna organización social puede arrebatárselos; gratuidad, asumida como la no sujeción a restricciones económicas para el acceso, y equidad, basada en el principio de justicia social, propio de la postura marxista-igualitarista, la cual predica: "a cada cual según su necesidad y de cada cual según su capacidad" (39).

\section{Avanzar hacia la consolidación de sistemas} universales de salud. La estructuración de sistemas universales de salud, con la visión de la salud como derecho humano fundamental, es compartida con el movimiento de la MSL y SC y la perspectiva de justicia social del igualitarismo, que asumen como función fundamental del sistema de salud, garantizar el derecho fundamental a la salud, con universalidad, integralidad, calidad, participación social, interculturalidad, solidaridad y acción sobre la determinación social de la salud. Aquí, se reconoce una visión alternativa de los sistemas de salud, donde el financiamiento no es una función del sistema de salud, sino un prerrequisito para su funcionamiento, y debe ser garantizado por el Estado. Se enfatiza que no es función del sistema de salud obtener el financiamiento fuera del Estado, promoviendo seguros privados, sino recaudar, distribuir adecuadamente y administrar con eficiencia los recursos financieros públicos (38).

En esta visión se asume como prioridad el desarrollo de nuevos modelos de atención, de carácter integral, con organización de redes basadas en el fortalecimiento de la atención primaria en salud, de predominio público, que enfaticen la promoción de la salud, la interculturalidad, la participación social, la acción sobre los determinantes sociales, el alcance de la universalidad y la eliminación de la exclusión social en salud (33).

Es así como los sistemas universales de salud tienen un compromiso con el derecho a la salud, la equidad y la solidaridad. Deben estar soportados en una concepción integral de la salud que incluya las dimensiones biológicas, psicológicas, sociales y ambientales, y que además integre sectores, instituciones, 
disciplinas y organizaciones comunitarias cuyo objetivo sea un abordaje de la salud basado y orientado hacia la justicia social, la equidad, el desarrollo humano, la dignidad y la calidad de vida (40).

De esta manera, para que efectivamente se avance en la configuración de políticas públicas y de un sistema de salud garante del derecho a la salud, entendido este más allá de la simple atención médica a la enfermedad, se requiere un consenso con la mayoría de las fuerzas políticas, del sector privado, de las organizaciones de la sociedad civil y la de Academia, entre otros.

\section{Conclusiones}

En este ensayo se presentan tres corrientes de pensamiento, relacionadas con la concepción de justicia social: la liberal radical u ortodoxa, la liberal igualitarista o redistributiva y la marxista o igualitarismo, y a partir de ellas se identifican algunas de las implicaciones que se han presentado en los sistemas de salud. Es así como, cada Estado construye el sistema de salud que responde a sus criterios políticos e ideológicos, para desde allí enfrentar y tratar de resolver los problemas de salud y enfermedad de la población.

De esta manera, la concepción de justicia social sobre la que se soportan los sistemas de salud infiltra las estructuras económicas, políticas y sociales. En consecuencia, cada país edifica un sistema de salud acorde con los conceptos, principios y valores que sustentan esa sociedad.

Por lo tanto, se propone una construcción alternativa a los sistemas de salud desde el principio del derecho a la salud como elemento de justicia, lo que implica partir de la convicción de la necesitad de estructurar sistemas de salud donde prime el bien común, la rentabilidad social, la ética ligada a proteger la vida y un modelo de atención que llegue a los espacios cotidianos de la vida. Para hacer esto posible, se requieren sistemas de salud que estén fundados en la declaración constitucional del derecho a la salud como un derecho humano fundamental, en la ampliación de la intervención del Estado, relacionada con la producción y distribución de los medios de la salud y en la consolidación de una apertura de espacios para una amplia, efectiva y dinámica participación de las personas y las organizaciones sociales en los procesos de planeación, toma de decisiones, control y vigilancia del sistema. Asimismo, es indispensable garantizar el acceso universal al sistema de salud, como una forma de equidad, que no esté determinado por el nivel de ingreso, ocupación en la economía o pertenencia a un sistema de aseguramiento público o privado.

Para construir y reconstruir sistemas de salud equitativos y con enfoque diferencial, se debe recurrir al debate político, y dejar de lado el tecnocrático, centrado en los servicios de salud, hasta llevarlo al ámbito de la justicia social. La política es una posibilidad de concretar trasformaciones. El derecho a la salud es una construcción entre el Estado y el ciudadano, como un reconocimiento de las igualdades de las personas; por lo tanto, es necesario rescatar la política como motor transformador de las realidades humanas, generando otra conciencia que potencie la acción para el disfrute pleno de la salud como un derecho humano.

La política debe hacer posible lo que es necesario.

\section{Agradecimientos}

$\mathrm{Al}$ apoyo financiero brindado por la Estrategia de Sostenibilidad 2013-2014 del Comité para el Desarrollo de la Investigación (CODI) 
de la Universidad de Antioquia, Grupo de Investigación Gestión y Políticas en Salud.

A los profesionales del grupo de apoyo del Centro de Investigación de la Facultad Nacional de Salud Pública de la Universidad de Antioquia: María Coral Correa y Yeferson Castaño Pineda, por su incondicional apoyo durante la construcción y culminación del artículo.

\section{Referencias bibliográficas}

1. Vega R. Dilemas éticos contemporáneos en salud: el caso colombiano desde la perspectiva de la justicia social. Rev Gerenc Polít Salud. 2002; 1 (2): 41-65.

2. Hobbes T, Leviatán O. La materia, forma y poder de una república eclesiástica y civil, quinta edición. San Juan de Puerto Rico: Editorial de la Universidad de Puerto Rico; 1968.

3. Locke J. Ensayo sobre el gobierno civil [internet]. México: Librería Porrúa; 2003 [acceso: 15 de diciembre del 2014]. Disponible en: http://juango.es/ lockeensayogobierno.pdf. In.

4. Hernández M. Seminario "La discusión determinantes o determinación social de la salud desde una reflexión de la justicia social', Unidad 7 'Desigualdad, inequidad e injusticia en el debate actual en salud'. Doctorado en Salud Pública, Facultad Nacional de Salud Pública, Medellín: Universidad de Antioquia; 2012.

5. Nozick R. Anarchy, State, and Utopia. Nueva York: Basic Books; 1974.

6. Borrero YE. Derecho a la salud, justicia sanitaria y globalización: un debate pendiente. Rev Fac Nac Salud Pública. 2011; 29 (3): 299-307.

7. Álvarez LE. Introducción. En: Facultad Nacional de Salud Pública. Pensamiento en salud pública. El derecho a la salud. Medellín: Editorial Universidad de Antioquia; 2001.

8. Uribe M. La justicia distributiva en la concepción libertaria de Nosick y Hayek. Rev Agenda, Universidad de los Andes. 2000; 151-63.

9. Hernández M. Preguntas a las bases morales de la reforma a la seguridad social en Colombia. En: VII Congreso Latinoamericano de Medicina Social, Buenos Aires, 1997.

10. Franco A. Tendencias y teorías en salud pública. Rev Fac Nac Sal Púb. 2006; 24 (2): 109-30.

11. Asociación Latinoamericana de Medicina Social. Taller latinoamericano de determinantes sociales de la salud 2003 [internet] [acceso: 15 de diciembre del 2014]. Disponible en: http://www.alames.org/documentos/ponencias.pdf.

12. Akin J, Birdsall N, De Ferranti D. Financiamiento de los servicios de salud en los países en desarrollo: Programa de reformas. Washington: Banco Mundial; 1990.

13. Hernández M, Hernández A, Perdomo A, Rodríguez $\mathrm{O}$, Torres $\mathrm{M}$, Vega $\mathrm{M}$, et al. La superación de la exclusión social en la región andina de América Latina: debates e implicaciones. Informe final del grupo Colombia-Nodo conjunto regional para América Latina para la red de Conocimiento sobre Exclusión Social (SEKN) de la Organización Mundial de la Salud (oms) (en prensa).

14. Navarro V. The Political Economy of Social Inequalities. Consequences for Health and Quality of Life. Nueva York: Baywood; 2002.

15. Hernández M. Problemas éticos: desigualdad, inequidad e injusticia. En: Taller Latinoamericano de Determinantes Sociales de la Salud, organizado por la Asociación Latinoamericana de Medicina Social (Alames), México, 2008.

16. Almeida C. Reforma del sector salud y equidad en América latina y el Caribe: conceptos, agendas, modelos y algunos resultados de implementación. Rev Geren Polít Salud. 2005; 4 (9): 6-60.

17. Granda E. ¿A que llamamos salud colectiva, hoy? Rev Cubana Med Gen Integr. 2004; 30 (2).

18. Breilh J. Epidemiología crítica. Ciencia emancipadora e intercultural. Buenos Aires: Universidad Nacional de Lanus, Lugar Editorial; 2003.

19. World Health Organization. Health System Strengthening Strategy. Ginebra: wHо; 2006.

20. Organización Mundial de la Salud. Informe sobre la salud en el mundo 2000. Mejorar el desempeño de los sistemas de salud. Ginebra: oms; 2000.

21. Fleury S. A questão democrática na saúde. Saúde e democracia: a luta do Cebes. San Pablo: Lemos; 1997.

22. Brehil J. Una perspectiva emancipadora de la investigación y acción, basada en la determinación social de la salud [internet]. México: Alames - Universidad Autónoma Metropolitana: 2011 [acceso: 17 de diciembre del 2014]. Disponible en: http://www.saludcolectivaunr.com.ar/docs/SeminarioBreilh03.pdf.

23. Banco Mundial. Informe sobre el Desarrollo Mundial 1993. Invertir en salud. Washington: Banco Mundial: 1993.

24. Muguerza J. La alternativa del disenso (en torno a la fundamentación ética de los derechos humanos). En: Peces-Barba G, editor. El fundamento de los derechos humanos. Madrid: Debate; 1989.

25. Rawls J. Teoría de la justicia. México: Fondo de Cultura Económica; 2006.

26. Gracia D. ¿Es un sistema justo de programas de salud? Principios para la asignación de recursos escasos. Boletín de la Oficina Sanitaria Panamericana (número especial sobre Bioética). 1991; 108 (5-6): 570-85.

27. The World Bank. Financing Health Services in Developing Countries. An Agenda for Reform. A World Bank Policy Study. Resumen en español: El financiamiento de los servicios de salud en los países en desarrollo. Boletín de la Oficina Sanitaria Panamericana. 1987; 103 (6): 695-709.

28. Organización Mundial de la Salud. Alocución del Director General a la 57a Asamblea Mundial de la Salud [internet].Washington: oms; 2004 [acceso: 19 de enero del 2015]. Disponible en: http://www.who. int/dg/lee/speeches/2004/wha57/es/.

29. Organización Mundial de la Salud, Commission on Social Determinants of Health. Commission on Social Determinants of Health. Towards a Concep- 
tual Framework for Analysis and Action on Social Determinants of Health. Ginebra: World Health Organization; 2005.

30. Marmot M. Social Causes of Social Inequalities in Health. En: Anand S, Sen A, Peter F, editores. Public Health, Ethics, and Equity. Oxford: Oxford University Press; 2004.

31. Organización Mundial de la Salud, Comisión sobre Determinantes Sociales de la Salud. Resumen analítico del informe final. Subsanar las desigualdades en una generación. Alcanzar la equidad sanitaria actuando sobre los determinantes sociales de la salud. Ginebra: Organización Mundial de la Salud; 2008.

32. Venkatapuram S. Global Justice and the Social Determinants of Health. Ethics Int Aff. 2010; 24 (2): 119-30.

33. Giovanella L, Feo O, Faria M, Tobar S. Sistemas de salud en Suramérica: desafíos para la universalidad la integralidad y la equidad. Rio de Janeiro: ISAGS, 2012.

34. Quevedo E, Hernández M. La articulación del conocimiento básico biológico y social en la formación del profesional de la salud: una mirada desde la Historia. En: Rodríguez MI, coordinador. Lo biológico y lo social. Su articulación en la formación de personal de salud. Washington: ops/oms, Serie Desarrollo de Recursos Humanos N 101; 1994.

35. Laurell AC. Para el estudio de la salud en su relación con el proceso de producción. En: Asociación Latinoamericana de Medicina Social. Taller Latinoame- ricano de Medicina Social. Medellín: Universidad de Antioquia-Alames; 1987.

36. Breihl J. Epidemiología: medicina, economía y política. México: Fontamara; 1986.

37. Breilh J. Addressing the Challenges of Social Determination of Health: a View from the South. Ponencia presentada en the Teleconference, University of Toronto, Dalla Lana School of Public Health, febrero del 2013. Quito: Universidad Andina Simón Bolívar; 2013.

38. Torres M. Alames expresión organizativa de la Medicina Social en América Latina. Rev Medicina Social. 2007; 2 (3): 139-44.

39. Currea V, Hernández M, Paredes N. Programa Venezolano de Educación-Acción en Derechos Humanos "La salud como derecho. Marco nacional e internacional de protección al derecho humano a la salud”. En : Currea V, Hernández M, Paredes N. La salud está grave. Una visión desde los derechos humanos. Bogotá: Plataforma Colombiana de Derechos Humanos, Democracia y Desarrollo; 2000.

40. Molina G, Ramírez A, Ruiz M. Tensiones en las decisiones en la salud pública en el sistema de salud colombiano: El bien común en confrontación con los intereses y prácticas particulares [internet]. Medellín: Universidad de Antioquia Pulso y Letra Editores; 2013 [acceso: 7 de febrero del 2015]. Disponible en: http:// www.udea.edu.co/portal/pls/portal/docs/1/1116516.pdf. 